\title{
Pharmacoethics and Pregnancy: Overcoming the drug orphan stigma
}

\author{
Jamil Kazma ${ }^{1}$, Johannes van den Anker (NO FURTHER ASSIGNMENTS) ${ }^{2}$, and Homa \\ Ahmadzia ${ }^{1}$ \\ ${ }^{1}$ The George Washington University \\ ${ }^{2}$ Children's National Medical Center
}

September 24, 2021

\begin{abstract}
There is paucity of evidence to support clinical decision making and counseling related to medication use in pregnancy. Despite multiple efforts from legislative bodies and advocacy groups, the inclusion of pregnant women in clinical drug trials assessing efficacy and safety remains scarce. Pregnancy can be complicated by multiple co-morbidities that require pharmacological intervention; these interventions primarily target the pregnant women but also sometimes have secondary effects for the fetus. The U.S. Food and Drug Administration has issued multiple guidance documents on incorporating pregnant women in clinical trials to aid pharmaceutical companies in designing a protocol to ensure safety and adherence to ethical standards. Advances in pediatric pharmacology studies provide lessons for researchers on the best practice of designing clinical trials with inclusion of patients from special populations. In this review, we present the status of pregnant women in clinical trials, highlighting the ethical stigma and possible future directives.
\end{abstract}

\section{Pharmacoethics and Pregnancy: Overcoming the drug orphan stigma}

Jamil M. Kazma ${ }^{1}$, John Van Den Anker ${ }^{2,3}$, Homa K. Ahmadzia ${ }^{4}$

${ }^{1}$ Department of Obstetrics \& Gynecology, The George Washington University School of Medicine and Health Sciences, Washington D.C., USA

${ }^{2}$ Division of Clinical Pharmacology, Children's National Hospital, Washington D.C., USA

${ }^{3}$ Division of Pediatric Pharmacology and Pharmacometrics, University Children's Hospital Basel, University of Basel, Switzerland

${ }_{4}$ Division of Maternal-Fetal Medicine, Department of Obstetrics \& Gynecology, The George Washington University School of Medicine and Health Sciences, Washington D.C., USA

*Corresponding author: Homa K. Ahmadzia, MD Division of Maternal Fetal Medicine Department of Obstetrics and Gynecology The George Washington University School of Medicine and Health Sciences 2100 Eye Street, NW, 20037 Phone: 202-741-2500 Email: hahmadzia@mfa.gwu.edu

ORCID Jamil M. Kazma 0000-0002-6447-5473 Homa K. Ahmadzia 0000-0003-0341-1086 John Van Den Anker 0000-0002-3585-321X

Running head: Pharmacoethics and Pregnancy Keywords: Pregnancy, Pharmacology, Teratogenicity, Fetotoxicity

Word count:Abstract 148, Manuscript 3015 words Number of tables and figures: 2 tables, 1 figures (max 8) Number of references: 66 


\title{
Conflict of interest
}

None of the authors have any conflict of interest to disclose

Funding statement

HKA was supported by K23HL141640.

\begin{abstract}
There is paucity of evidence to support clinical decision making and counseling related to medication use in pregnancy. Despite multiple efforts from legislative bodies and advocacy groups, the inclusion of pregnant women in clinical drug trials assessing efficacy and safety remains scarce. Pregnancy can be complicated by multiple co-morbidities that require pharmacological intervention; these interventions primarily target the pregnant women but also sometimes have secondary effects for the fetus. The U.S. Food and Drug Administration has issued multiple guidance documents on incorporating pregnant women in clinical trials to aid pharmaceutical companies in designing a protocol to ensure safety and adherence to ethical standards. Advances in pediatric pharmacology studies provide lessons for researchers on the best practice of designing clinical trials with inclusion of patients from special populations. In this review, we present the status of pregnant women in clinical trials, highlighting the ethical stigma and possible future directives.
\end{abstract}

\section{Introduction}

Pregnant women are poorly represented in clinical drug trials. Around $80 \%$ of pregnant women receive medication during pregnancy, most of which are used in an off-label manner and lack information on potential teratogenicity [1]. In fact, from 2000 to 2010, the U.S. Food and Drug Administration (FDA) approved 172 new drugs, $98 \%$ of which lack data on teratogenicity and $73 \%$ had no data on safety for use during pregnancy [2]. Many women with pre-existing conditions such as asthma, chronic hypertension, and diabetes might be taking medication prior to conception. In addition, the physiologic changes in pregnancy can exacerbate existing medical conditions or induce new medical conditions that require treatment. This highlights the importance of the availability of safety data on drugs prescribed during pregnancy. To complicate matters further, the doses of drugs used during pregnancy are extrapolated from clinical drug investigations performed in men and non-pregnant women and/or from animal models, raising questions about the pharmacokinetics and pharmacodynamics of these drugs during the different stages of pregnancy [3].

One of the landmark events in obstetric pharmacology was the thalidomide disaster that occurred in the 1960s. This drug, used for morning sickness in pregnant women, was found to be teratogenic, causing devastating skeletal deformities in prenatally exposed fetuses [4]. Another drug, diethylstilbestrol, a synthetic estrogen, was initially prescribed to women with threatened pregnancy loss and then was marketed as routine for prophylaxis of possible pregnancy loss for all pregnancies during the 1950s [5]. Twenty years later after continued and extensive use, a small study showed an increased risk of clear cell carcinoma of the vagina in females born to diethylstilbestrol-exposed pregnant women among other comorbidities before regulatory action was taken to curtail its use [6]. Historically, after the thalidomide and diethylstilbestrol negative outcomes, the US FDA has excluded pregnant women from phase 1 and phase 2 trials in 1977 over concerns for the safety of administering drugs in pregnancy, after which the pharmaceutical companies extended this exclusion into phase 3 and phase 4 [7]. These examples among many others underscore the importance of risk and benefit assessment of medication use during pregnancy. However, the current evidence to support this assessment is sparse. In this review, we aim to highlight a) the need for inclusion of pregnant women in clinical trials, b) what legislative actions were taken to curtail obstacles for their inclusion, and c) possible solutions for clinical pharmacology researchers.

\section{The Ethical Dilemma across history}

Prioritizing protection of the fetus was an important factor that prevented pregnant women to participate in clinical drug trials. Several federal regulations now request clarification on inclusion criteria by defining whether the fetus or the pregnant woman are being targeted, and whether the study aims to highlight 
therapeutic or non-therapeutic outcomes [8]. In 1974, after the devastating thalidomide disaster, congress asked the newly established National Commission for the Protection of Human Subjects of Biomedical and Behavioral Research to make recommendations for the conduct of research involving pregnant women and fetuses (Federal regulations at subpart B of 45 CFR 46) [9]. Dr. Kelsey, who was the primary reviewer of the thalidomide application assigned by the FDA, delayed the approval of thalidomide over concerns of adverse effects and the drug was never approved in the United States [10]. The aim of the recommendations thus was to protect the fetus from unnecessary harm. More than twenty years later and after much deliberation, the wording was changed in 2001 to include a more proscriptive approach; stating that pregnant women or fetuses may be involved in research if all of 10 conditions are met (Table 1). In 2002, a registry for reporting adverse effects was established. In 2004, the FDA developed the guidance on pharmacokinetic (PK) studies in pregnancy, and, in 2005, guidance was provided on clinical lactation studies and pregnancy [11]. In 2009, the Second Wave Initiative was launched aiming to systematically address the knowledge gap on treatment of pregnant women in a collaborative manner [12]. The FDA has also addressed drug labeling to include risk summary and clinical consideration in an effort to improve patient care decision and counseling under the Pregnancy and Lactation Labeling Rule (PLLR) [7]. This was first proposed in 2008 with the revised version put in action in 2014. While much has been done to support the moral imperative of including pregnant women, much more needs to be done to ensure that we provide pregnant women with beneficence, autonomy, and justice.

\section{Pitfalls of Maternal Physiology and Placental Transfer}

Substantial changes in maternal physiology complicate the extrapolation of the safety profile and dosing of drugs. Changes in the cardiovascular, renal, and gastrointestinal systems affect the absorption, distribution and excretion of certain drugs given to the pregnant woman during pregnancy [13]. There is no doubt that drug pharmacokinetics are different in pregnancy and non-pregnant state and, ideally, drug properties should be studied in every trimester and in the postpartum period. However, these trials are challenging to conduct, and the information is scarce, which is why researchers often rely on opportunistic studies in which patient are already receiving the therapeutic agent in question $[14,15]$. In addition to that, the placenta was thought to be an impenetrable barrier that protected the fetus from harmful agents including medication. However, after the thalidomide incident, challenges have been unearthed to explore mechanisms of transfer of compounds across the lipid membrane [16]. Several mechanisms have been proposed to explain the drug transfer across the placenta including simple diffusion, facilitated diffusion, pinocytosis, and active transport. Ongoing research is crucial in identifying potential drugs that follow the trajectory of maternal to fetal transfer, and the molecular characteristics of compounds. Uncharged lipophilic drugs tend to transfer readily [17], whereas size does not usually limit transfer as most drugs have a molecular weight of less than 500 Daltons [18].Insulin and enoxaparin are two examples of drug transfers that are limited due to size [19, 20]. Human placental drug transfer studies are often limited to drugs given near time of delivery. This limitation to study design led to development of ex vivo perfused human placental models which represented a non-invasive and effective method of studying transplacental transfer [21].

\section{Importance of data on drugs for pregnant women}

\section{Physician Counseling}

Counseling is a key concept in any patient encounter, particularly when it comes to initiate new therapies during pregnancy as it helps consolidate a patient-centered practice. In fact, informing patients about the indication, dosage, regimen, side effect(s) and alternatives can improve adherence and limit therapeutic failure, or help to recognize adverse effects that otherwise might result in unnecessary diagnostic tests and hospitalization [22]. It is imperative for providers during the prenatal visit to obtain a thorough history about medical problems and medications used to treat them, and to screen for herbal medicine use in pregnancy which is particularly important as the use of complementary and alternative medicine is high globally [23]. Some women might have stopped taking their medications prior to the first visit with a provider after finding out they are pregnant. This action can sometimes lead to deleterious consequences. For example, women treated with SSRIs for depression who have discontinued the medication of fear of fetal concern may have 
relapses and suicidal ideation [24]. On the other hand, counseling may help prevent unnecessary pregnancy termination because of perceived high fetal risk not knowing the accurate extent of the risk in question due to lack of proper information [25].

During the counseling, the provider must also distinguish between teratogenicity which entails structural abnormalities to the fetus in the first trimester and fetotoxicity referring to functional damage later in pregnancy [26]. While the teratogenicity profile of some drugs has been established through prior animal studies, detecting fetotoxicity requires more research particularly as some effects pertaining to neurodevelopment might not be evident until childhood. Women prefer to seek information about medication use in pregnancy directly from their healthcare providers. However, the challenge arises when there is lack of evidence-based data to appropriately display a risk-benefit assessment for the patient to decide [22, 27]. Database such as

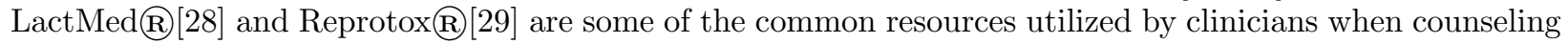
patients, but these databases can often give inconclusive recommendations given the paucity of evidence available.

\section{Treating life-threatening diseases and those associated with morbidities}

There is a considerable increase in maternal comorbidity including obesity, hyperlipidemia, diabetes, and hypertension in recent years worldwide. Many of these comorbid conditions have been linked to higher rates of pregnancy-related morbidity and mortality [30]. Asthma for example can be common in pregnancy and in some cases worsens due to physiological changes. In one study, it has been shown that there has been a reduction in prescriptions of asthma medication during the first trimester of pregnancy [31,32]. However, it is important to control asthma symptoms during pregnancy despite the safety profile of some medications used. Uncontrolled asthma can lead to complications including preeclampsia, preterm delivery and low birth weight [33]. This is a compelling example where risks of uncontrolled asthma outweigh potential risks of neonatal fetotoxicity from exposure. Another example are oral steroids like prednisone. Prior observational studies have reported cleft lip and cleft palate when prednisone was used early in pregnancy but those results were not consistent over time [34]. Table 2 highlights controversial medications that need additional counseling given their possible or known adverse effects on the fetus. There is much controversy in interpreting population-based studies and animal studies when it comes to teratogenicity and fetotoxicity. Some drugs that showed adverse effects in animal models were not matched when studying human population and vice versa. Statins, which are now being investigated as potential drugs to prevent preeclampsia have been previously contraindicated in pregnancy. However, a recent meta-analysis including 16 human studies showed no relationship between statins and teratogenicity [35]. More recently, the FDA has requested removal of the contraindication of statins in pregnancy [36].

Improving the infrastructure of research seems to be something that would benefit the moral imperative to incorporate pregnant women into clinical drug trials. Pharmacoepidemiologic studies are limited by the fact that often disease and severity are related to exposure and adverse outcome and that systematic bias is not usually accounted for when the pharmacologic exposure or the disease itself was the cause of fetotoxicity. For example, autoimmune diseases like rheumatoid arthritis, inflammatory bowel disease or lupus might exacerbate during pregnancy. In these diseases it has been noted that there is an increased risk of low birth weight, preterm birth and associated morbidities with current treatment options, but whether this is in an effect of the treatment per se or the disease itself is hard to decipher [37-39].

\section{The effect of the regulatory bodies on clinical trials in pregnancy}

Pregnant women have previously been categorized as a "vulnerable population' with special consideration enforced by regulatory bodies when including them in research. The American College of Obstetrics and Gynecology instead recommended that pregnant women be categorized under "scientifically complex," and recently the Common Rule, the federal policy for the protection of human subjects, has been revised in 2019 to remove pregnant women from the vulnerable population category [40]. However, despite that, some institutional review boards (IRB) may still feel reluctant given that there is no practical guide to address the risk and benefits of enrolling pregnant women into clinical trials [41]. Two important steps to curtail this is 
to involve experts in the field of maternal-fetal medicine and obstetrics pharmacology in board meetings and to require justification for exclusion of pregnant women and that this justification may be questioned during review [42]. IRB interpretation of the regulatory process has some flaws [11], particularly when it comes to the wording of "minimal risk of the fetus." When submitting to the IRB of the academic institution or other regulatory bodies, researchers may present preclinical and animal studies corroborating the risk to the fetus, but with concern that these studies are evaluated by the IRB regulatory staff and due to the dearth of available data may not be sufficiently supportive or convincing[11]. For that reason, there is wide agreement to clarify regulations for enrolling pregnant women in trials and develop practical guidelines that can be universally implemented [15].

The FDA previously had regulatory rules in place that restricted inclusion of pregnant women in clinical trials, citing that woman should only be included if there is direct benefit to the woman or fetus with minimal risk or if risk is solely related to the intervention in question [43]. In their revised guideline in 2018, they have recommended excluding pregnant women from phase 1 and phase 2 trials and allow enrollment later. Federal regulations require investigators to consider the interest of the pregnant woman and fetus, raising the ethical question of whether the fetus is considered a patient. One argument involves a dependent moral status to be deemed on the fetus, which is based on the expectation of whether the fetus is to achieve the moral status of becoming a child and a person [44]. It is only when the pregnant woman considered the previable fetus a patient and therefore invoke the dependent moral status, then the healthcare provider and patient should have a thorough discussion about the beneficence of protecting the fetus from harm.

Pharmaceutical companies have long feared including pregnant women in clinical trials even during the phase 3 and phase 4 of the process. In response to that, the FDA has issued a guidance for industries to better design clinical trials. It highlights emphasis on pharmacokinetic and pharmacodynamic studies of drugs in pregnant women, particularly if that population is to benefit from the drug. In addition, in 2002, the FDA also issued a guidance on reporting adverse effects of medication in pregnancy and that surveillance should not be limited to the post-marketing phase. The recent events of the COVID-19 pandemic have highlighted the deficiency and reluctance posed on including pregnant women in trials. In a recent analysis, it has been shown that pregnant women have been excluded from the therapeutic clinical trials involving the COVID-19 infection, despite most medications used showing low or non-significant safety concerns, except for remdesivir [45]. This only increases the concerns that despite guidance and call to action initiatives, there is no legal framework to enforce its implementation.

\section{Extrapolating innovations from pediatric clinical trials}

While neonates and children have been lumped with pregnant women under the umbrella of vulnerable populations, numerous advances have led to success in conducting pediatric clinical trials [46]. Quality of clinical data in pediatrics has stemmed from the fact that investigators tend to include multiple drugs in a single protocol, extensive pharmacokinetic and pharmacodynamic modeling and incorporation of multiple sites [47]. As for legislative processes, the Best Pharmaceuticals for Children Act (BPCA) and Pediatric Research Equity Act (PREA) contributed to the development of drug trials in the pediatric population. The BPCA provided incentive for drug development and PREA enforces pediatric studies to be conducted of medication that would help the health of children. Incentives included exclusive marketing by manufacturers for an additional six month for conducting pediatric-focused studies, which can translate to up to 500 million dollars in revenue for each drug [48]. Unfortunately, these do not yet apply to the pregnant population.

Recent innovations in clinical trial design allowed the development and use of population-based pharmacokinetic and pharmacodynamic modeling to better understand the mechanism of drugs within the physiologic milieu $[49,50]$. These advances will likely help optimize selected drug doses and understand interaction with tissues in an attempt to minimize unwanted adverse effects to the fetus. These models have been described by Mendes and Zhang [49, 50]. Population pharmacokinetics-pharmacodynamics studies have been used in pediatric clinical trials and have been found to be very helpful as they incorporate drug properties, physiologic variables, and target tissues to determine effect [51]. Despite its success in pediatric populations, its use in the pregnant population seems underutilized. 
A multi-disciplinary collaboration is necessary to optimally design and conduct clinical drug trials in pregnant women. For example, if a rheumatologist wishes to conduct a trial on effective treatment of rheumatoid arthritis flare and include pregnant women, a collaboration with maternal-fetal medicine and neonatology specialists should be considered to optimize outcome of the study and avoid adverse effect of treatment or disease on the fetus.

\section{A complex twist: treating the fetus through the pregnant woman}

Some fetal conditions are treated through transplacental transfer of drugs adding a complex layer on the safety of drugs. While the drug is administered to the pregnant woman and has known maternal side effects, the primary indication is to treat the underlying fetal condition. One important condition is fetal arrythmia (i.e. supraventricular tachycardia) in which studies have shown benefit in administering digoxin, sotalol, and flecainide among other antiarrhythmic medications [52]. In this unique circumstance often added maternal monitoring by electrocardiogram is advised to balance maternal/fetal well-being. Another example is prevention of congenital toxoplasmosis. Spiramycin, a well-known macrolide, has shown to decrease transmission of toxoplasmosis in a seropositive pregnant woman by $60 \%$. Due to its chemical properties, it tends to concentrate in the placenta and rarely transfer to the fetus mitigating adverse effects and fetotoxicity [53].

\section{Conclusion}

The Belmont Report originally published in 1979 enforces the adherence to the basic ethical principles in research including beneficence, justice, and respect to persons. We need a call to action by all stakeholders (i.e. legislative agencies, pharmaceutical companies, funders and academic researchers) to prioritize including pregnant women in clinical trials. It helps not only make a better risk-benefit assessment, but also help prevent adverse effects in the fetus or the pregnant woman when it is medically needed to give medications. We should strengthen the pregnant woman's autonomy to be able to participate in clinical trials if she wishes and that this should not be a predicament; at the same time monitoring for fetotoxicity should be standardized and required in clinical research. If pregnant women are not included in future drug research studies, they will lag behind in terms of receiving benefits of therapeutic advancement compared to the general population. Learning from pediatric drug advances, clinical research involving pregnant women has a promising road ahead.

\section{References}

1. Grzeskowiak LE, Mol BW. Off-label prescribing in pregnancy-a case of risky business or business as usual? Eur J Obstet Gynecol Reprod Biol 2015; 187: 78-9.

2. Adam MP, Polifka JE, Friedman JM. Evolving knowledge of the teratogenicity of medications in human pregnancy. Am J Med Genet C Semin Med Genet 2011; 157C: 175-82.

3. van der Graaf R, van der Zande ISE, den Ruijter HM, Oudijk MA, van Delden JJM, Oude Rengerink K, Groenwold RHH. Fair inclusion of pregnant women in clinical trials: an integrated scientific and ethical approach. Trials 2018; 19: 78.

4. Somers GS. Thalidomide and congenital abnormalities. Lancet 1962; 1: 912-3.

5. Troisi R, Hatch EE, Titus L. The Diethylstilbestrol Legacy: A Powerful Case Against Intervention in Uncomplicated Pregnancy. Pediatrics 2016; 138: S42-S44.

6. Herbst AL, Scully RE. Adenocarcinoma of the vagina in adolescence. A report of 7 cases including 6 clear-cell carcinomas (so-called mesonephromas). Cancer 1970; 25: 745-57.

7. Sheffield JS, Siegel D, Mirochnick M, Heine RP, Nguyen C, Bergman KL, Savic RM, Long J, Dooley KE, Nesin M. Designing drug trials: considerations for pregnant women. Clin Infect Dis 2014; 59 Suppl 7: S437-44.

8. Briggs GG, Freeman RK, Tower CV. Drugs in Pregnancy and Lactation: A Reference Guide to Fetal and Neonatal Risk. 12 Editon. Baltimore: Lippincott Williams \& Wilkins (LWW), 2021. 
9. Regulations UCoF. Protection of human subjects. 45 CFR 46, Subpart B. In, 2009.

10. Ances BM. New concerns about thalidomide. Obstet Gynecol 2002; 99: 125-8.

11. Blehar MC, Spong C, Grady C, Goldkind SF, Sahin L, Clayton JA. Enrolling pregnant women: issues in clinical research. Womens Health Issues 2013; 23: e39-45.

12. Lyerly AD, Little MO, Faden R. The second wave: Toward responsible inclusion of pregnant women in research. Int J Fem Approaches Bioeth 2008; 1: 5-22.

13. Kazma JM, van den Anker J, Allegaert K, Dallmann A, Ahmadzia HK. Anatomical and physiological alterations of pregnancy. J Pharmacokinet Pharmacodyn 2020; 47: 271-85.

14. Torres-Vergara P, Escudero C, Penny J. Drug Transport at the Brain and Endothelial Dysfunction in Preeclampsia: Implications and Perspectives. Front Physiol 2018; 9: 1502.

15. Lo WY, Friedman JM. Teratogenicity of recently introduced medications in human pregnancy. Obstet Gynecol 2002; 100: 465-73.

16. Vargesson N. Thalidomide-induced teratogenesis: history and mechanisms. Birth Defects Res C Embryo Today 2015; 105: 140-56.

17. Griffiths SK, Campbell JP. Placental structure, function and drug transfer. Bja Educ 2015; 15: 84-89.

18. Mirkin BL. Perinatal pharmacology: placental transfer, fetal localization, and neonatal disposition of drugs. Anesthesiology 1975; 43: 156-70.

19. Dimitrakakis C, Papageorgiou P, Papageorgiou I, Antzaklis A, Sakarelou N, Michalas S. Absence of transplacental passage of the low molecular weight heparin enoxaparin. Haemostasis 2000; 30: 243-48.

20. Desoye G, Hofmann HH, Weiss PAM. Insulin Binding to Trophoblast Plasma-Membranes and Placental Glycogen-Content in Well-Controlled Gestational Diabetic Women Treated with Diet or Insulin, in WellControlled Overt Diabetic-Patients and in Healthy Control Subjects. Diabetologia 1992; 35: 45-55.

21. Brownbill P, Sebire N, McGillick EV, Ellery S, Murthi P. Ex Vivo Dual Perfusion of the Human Placenta: Disease Simulation, Therapeutic Pharmacokinetics and Analysis of Off-Target Effects. Methods Mol Biol 2018; 1710: 173-89.

22. Devkota R, Khan GM, Alam K, Sapkota B, Devkota D. Impacts of counseling on knowledge, attitude and practice of medication use during pregnancy. BMC Pregnancy Childbirth 2017; 17: 131.

23. Kennedy DA, Lupattelli A, Koren G, Nordeng H. Herbal medicine use in pregnancy: results of a multinational study. BMC Complement Altern Med 2013; 13: 355.

24. Cohen LS, Altshuler LL, Harlow BL, Nonacs R, Newport DJ, Viguera AC, Suri R, Burt VK, Hendrick V, Reminick AM, Loughead A, Vitonis AF, Stowe ZN. Relapse of major depression during pregnancy in women who maintain or discontinue antidepressant treatment. JAMA 2006; 295: 499-507.

25. Koren G, Pastuszak A. Prevention of unnecessary pregnancy terminations by counselling women on drug, chemical, and radiation exposure during the first trimester. Teratology 1990; 41: 657-61.

26. Henderson E, Mackillop L. Prescribing in pregnancy and during breast feeding: using principles in clinical practice. Postgrad Med J 2011; 87: 349-54.

27. Sun D, Hutson JR, Garcia-Bournissen F. Drug therapy during pregnancy. Br J Clin Pharmacol 2020.

28. Tomasulo P. LactMed-new NLM database on drugs and lactation. Med Ref Serv Q 2007; 26: 51-8.

29. Fitzpatrick RB. REPROTOX: an information system on environmental hazards to human reproduction and development. Med Ref Serv Q 2008; 27: 73-80. 
30. Hayes DK, Robbins CL, Ko JY. Trends in Selected Chronic Conditions and Related Risk Factors Among Women of Reproductive Age: Behavioral Risk Factor Surveillance System, 2011-2017. J Womens Health (Larchmt) 2020; 29: 1576-85.

31. National Heart L, Blood I, National Asthma E, Prevention Program A, Pregnancy Working G. NAEPP expert panel report. Managing asthma during pregnancy: recommendations for pharmacologic treatment2004 update. J Allergy Clin Immunol 2005; 115: 34-46.

32. Zetstra-van der Woude PA, Vroegop JS, Bos HJ, de Jong-van den Berg LT. A population analysis of prescriptions for asthma medications during pregnancy. J Allergy Clin Immunol 2013; 131: 711-7.

33. Murphy VE, Gibson PG. Asthma in pregnancy. Clin Chest Med 2011; 32: 93-110.

34. Fraser FC, Fainstat TD. Production of congenital defects in the off-spring of pregnant mice treated with cortisone; progress report. Pediatrics 1951; 8: 527-33.

35. Karalis DG, Hill AN, Clifton S, Wild RA. The risks of statin use in pregnancy: A systematic review. J Clin Lipidol 2016; 10: 1081-90.

36. Adminstration FAD. FDA Drug Safety Communication. In, 2021.

37. Lateef A, Petri M. Managing lupus patients during pregnancy. Best Pract Res Clin Rheumatol 2013; 27: $435-47$.

38. Broms G, Granath F, Linder M, Stephansson O, Elmberg M, Kieler H. Birth outcomes in women with inflammatory bowel disease: effects of disease activity and drug exposure. Inflamm Bowel Dis 2014; 20: 1091-8.

39. Rom AL, Wu CS, Olsen J, Kjaergaard H, Jawaheer D, Hetland ML, Vestergaard M, Morch LS. Fetal growth and preterm birth in children exposed to maternal or paternal rheumatoid arthritis: a nationwide cohort study. Arthritis Rheumatol 2014; 66: 3265-73.

40. Rubin R. Addressing Barriers to Inclusion of Pregnant Women in Clinical Trials. JAMA 2018; 320: $742-44$.

41. Kaposy C. Presumptive Inclusion and Legitimate Exclusion Criteria. In: Clinical Research Involving Pregnant Women, edsBaylis F, Ballantyne A, Cham: Springer, 2016.

42. Payne P. Including Pregnant Women in Clinical Research: Practical Guidance for Institutional Review Boards. Ethics Hum Res 2019; 41: 35-40.

43. Administration. USFaD. Pregnant Women: Scientific and Ethical Considerations for Inclusion in Clinical Trials Guidance for Industry - Draft Guidance. In, 2018.

44. McCullough LB, Coverdale JH, Chervenak FA. A comprehensive ethical framework for responsibly designing and conducting pharmacologic research that involves pregnant women. Am J Obstet Gynecol 2005; 193: $901-7$.

45. Taylor MM, Kobeissi L, Kim C, Amin A, Thorson AE, Bellare NB, Brizuela V, Bonet M, Kara E, Thwin SS, Kuganantham H, Ali M, Oladapo OT, Broutet N. Inclusion of pregnant women in COVID-19 treatment trials: a review and global call to action. Lancet Glob Health 2021; 9: e366-e71.

46. Gonzalez D, Boggess KA, Cohen-Wolkowiez M. Lessons learned in pediatric clinical research to evaluate safe and effective use of drugs in pregnancy. Obstet Gynecol 2015; 125: 953-58.

47. Laughon MM, Benjamin DK, Jr., Capparelli EV, Kearns GL, Berezny K, Paul IM, Wade K, Barrett J, Smith PB, Cohen-Wolkowiez M. Innovative clinical trial design for pediatric therapeutics. Expert Rev Clin Pharmacol 2011; 4: 643-52. 
48. Benjamin DK, Jr., Smith PB, Murphy MD, Roberts R, Mathis L, Avant D, Califf RM, Li JS. Peerreviewed publication of clinical trials completed for pediatric exclusivity. JAMA 2006; 296: 1266-73.

49. De Sousa Mendes M, Hirt D, Vinot C, Valade E, Lui G, Pressiat C, Bouazza N, Foissac F, Blanche S, Le MP, Peytavin G, Treluyer JM, Urien S, Benaboud S. Prediction of human fetal pharmacokinetics using ex vivo human placenta perfusion studies and physiologically based models. Br J Clin Pharmacol 2016; 81: 646-57.

50. Zhang Z, Unadkat JD. Development of a Novel Maternal-Fetal Physiologically Based Pharmacokinetic Model II: Verification of the model for passive placental permeability drugs. Drug Metab Dispos 2017; 45: 939-46.

51. Benaboud S, Treluyer JM, Urien S, Blanche S, Bouazza N, Chappuy H, Rey E, Pannier E, Firtion G, Launay O, Hirt D. Pregnancy-related effects on lamivudine pharmacokinetics in a population study with 228 women. Antimicrob Agents Chemother 2012; 56: 776-82.

52. Alsaied T, Baskar S, Fares M, Alahdab F, Czosek RJ, Murad MH, Prokop LJ, Divanovic AA. FirstLine Antiarrhythmic Transplacental Treatment for Fetal Tachyarrhythmia: A Systematic Review and MetaAnalysis. J Am Heart Assoc 2017; 6.

53. Fung HB, Kirschenbaum HL. Treatment regimens for patients with toxoplasmic encephalitis. Clin Ther 1996; 18: 1037-56; discussion 36.

54. Di Carlo R, Pagnini G, Pelagalli GV. Effect of amitriptyline and butriptyline on fetal development in rats. J Med 1971; 2: 271-5.

55. Beyer BK, Guram MS, Geber WF. Incidence and potentiation of external and internal fetal anomalies resulting from chlordiazepoxide and amitriptyline alone and in combination. Teratology 1984; 30: 39-45.

56. Altshuler LL, Cohen L, Szuba MP, Burt VK, Gitlin M, Mintz J. Pharmacologic management of psychiatric illness during pregnancy: dilemmas and guidelines. Am J Psychiatry 1996; 153: 592-606.

57. Pastuszak A, Schick-Boschetto B, Zuber C, Feldkamp M, Pinelli M, Sihn S, Donnenfeld A, McCormack M, Leen-Mitchell M, Woodland C, et al. Pregnancy outcome following first-trimester exposure to fluoxetine (Prozac). JAMA 1993; 269: 2246-8.

58. Munk-Olsen T, Liu XQ, Viktorin A, Brown HK, Di Florio A, D'Onofrio BM, Gomes T, Howard LM, Khalifeh H, Krohn H, Larsson H, Lichtenstein P, Taylor CL, Van Kamp I, Wesseloo R, Meltzer-Brody S, Vigod SN, Bergink V. Maternal and infant outcomes associated with lithium use in pregnancy: an international collaborative meta-analysis of six cohort studies. Lancet Psychiat 2018; 5: 644-52.

59. Diav-Citrin O, Shechtman S, Tahover E, Finkel-Pekarsky V, Arnon J, Kennedy D, Erebara A, Einarson A, Ornoy A. Pregnancy Outcome Following In Utero Exposure to Lithium: A Prospective, Comparative, Observational Study. Am J Psychiat 2014; 171: 785-94.

60. Baldwin JA, Davidson EJ, Pritchard AL, Ridings JE. The reproductive toxicology of paroxetine. Acta Psychiatr Scand Suppl 1989; 350: 37-9.

61. Holzgreve W, Carey JC, Hall BD. Warfarin-induced fetal abnormalities. Lancet 1976; 2: 914-5.

62. Donofrio MT, Moon-Grady AJ, Hornberger LK, Copel JA, Sklansky MS, Abuhamad A, Cuneo BF, Huhta JC, Jonas RA, Krishnan A, Lacey S, Lee W, Michelfelder EC, Sr., Rempel GR, Silverman NH, Spray TL, Strasburger JF, Tworetzky W, Rychik J, American Heart Association Adults With Congenital Heart Disease Joint Committee of the Council on Cardiovascular Disease in the Y, Council on Clinical Cardiology CoCS, Anesthesia, Council on C, Stroke N. Diagnosis and treatment of fetal cardiac disease: a scientific statement from the American Heart Association. Circulation 2014; 129: 2183-242.

63. Reinisch JM, Simon NG, Karow WG, Gandelman R. Prenatal exposure to prednisone in humans and animals retards intrauterine growth. Science 1978; 202: 436-8. 
64. Bandoli G, Palmsten K, Smith CJF, Chambers CD. A Review of Systemic Corticosteroid Use in Pregnancy and the Risk of Select Pregnancy and Birth Outcomes. Rheum Dis Clin N Am 2017; 43: 489.

65. Harrod MJ, Sherrod PS. Warfarin embryopathy in siblings. Obstet Gynecol 1981; 57: 673-6.

66. Howe AM, Webster WS. The warfarin embryopathy: a rat model showing maxillonasal hypoplasia and other skeletal disturbances. Teratology 1992; 46: 379-90.

Table 1. The ten requirements for inclusion of pregnant women in clinical trials outlined by the FDA [43]

$1 \quad$ Where scientifically appropriate, preclinical studies, including studies on pregnant animals, and clinical studies, includi

2 The risk to the fetus is caused solely by interventions or procedures that hold out the prospect of direct benefit for the

3 Any risk is the least possible for achieving the objectives of the research;

4 If the research holds out the prospect of direct benefit to the pregnant woman, the prospect of a direct benefit both to

5 If the research holds out the prospect of direct benefit solely to the fetus then the consent of the pregnant woman and

6 Each individual providing consent under paragraph 4 or 5 of this section is fully informed regarding the reasonably for

$7 \quad$ For children as defined in $\S 46.402(\mathrm{a})$ who are pregnant, assent and permission are obtained in accord with the provisio

$8 \quad$ No inducements, monetary or otherwise, will be offered to terminate a pregnancy;

9 Individuals engaged in the research will have no part in any decisions as to the timing, method, or procedures used to

10 Individuals engaged in the research will have no part in determining the viability of a neonate.

Table 2. Some medications with controversial use in pregnancy

\begin{tabular}{ll}
\hline Medication & Condition \\
\hline Amitriptyline & Major depressive disorder; Migraine prophylaxis \\
Lithium & Bipolar disorder \\
Paroxetine; Fluoxetine & Major Depressive Disorder \\
Prednisone & Inflammatory conditions including rheumatoid arthritis, lupus nephritis, inflammatory bowel disea \\
Statins & Dyslipidemia \\
Warfarin & Thromboprophylaxis in patients with mechanical heart valve \\
\hline
\end{tabular}

Figure 1. Timeline of advancements in policy making towards drug trials in pregnancy

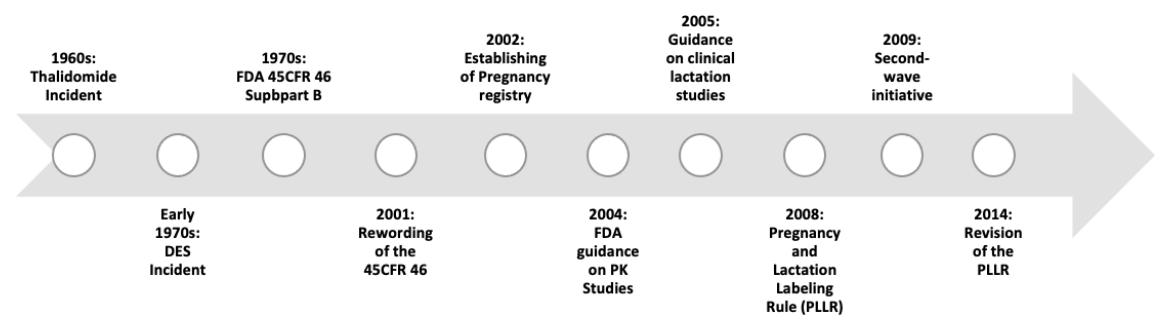

